\title{
Comparison of 1800 Robotic and Open Partial Nephrectomies for Renal Tumors
}

\author{
Adam Bezinque, $\mathrm{BS}^{1}$ and Brian R. Lane, $\mathrm{MD}, \mathrm{PhD}^{2,3}$ \\ ${ }^{1}$ Michigan State University College of Osteopathic Medicine, East Lansing, MI; ${ }^{2}$ Spectrum Health, Grand Rapids, MI; \\ ${ }^{3}$ Michigan State University College of Human Medicine, Grand Rapids, MI
}

Partial nephrectomy (PN) is the reference standard for renal tumors that are amenable to a kidney-sparing approach. With oncologic outcomes superior to thermal ablation and renal functional outcomes superior to radical nephrectomy, the number of situations in which $\mathrm{PN}$ is employed is growing. ${ }^{1,2}$ The outcomes of PN vary greatly with surgeon experience, variations in technique, and tumor complexity. Several techniques have been developed and modified during the past 30 years or more, with contemporary discussions addressing optimization of the oncologic, renal functional, and postoperative outcomes of PN. ${ }^{3}$

During the late 1990s, minimally invasive PN was pioneered using pure laparoscopic techniques. ${ }^{4,5}$ In the initial single-center series reported, laparoscopic PN (LPN) was found to be feasible and with acceptable morbidity when performed by master laparoscopic surgeons who were building upon the experience of prior experts' open surgical techniques. ${ }^{4-6}$ Comparison of results from these initial series of LPN with OPN performed well after the initial learning curve for this procedure revealed both advantages and disadvantages for LPN despite the somewhat imbalanced comparison. ${ }^{7}$ LPN offered the advantages of decreased operative time (median 3.3 vs. $4.3 \mathrm{~h}$; relative risk [RR]: 0.78 (95\% confidence interval [CI]: 0.75-0.81), $p<0.0001$ ), decreased operative blood loss (median 300 vs. $376 \mathrm{ml}$; RR: 0.80 (95\% CI: 0.74-0.83), $p<0.0001$ ),

(C) Society of Surgical Oncology 2016

First Received: 21 June 2016;

Published Online: 11 July 2016

B. R. Lane, MD, PhD

e-mail: brian.lane@spectrumhealth.org and shorter hospital stay (median 3.3 vs. 5.8 days; RR: 0.59 (95 \% CI: $0.56-0.61), p<0.0001$ ). In these 1800 patients, the disadvantages of LPN were longer warm ischemia time (median 31 vs. $20 \mathrm{~min}$; RR: 1.69 (95\% CI: 1.62-1.77), $p<0.0001)$, more urologic complications $(9.2$ vs. $5.0 \%$; odds ratio [OR]: 2.14 (95\% CI: 1.39-3.31), $p=0.0006$ ), and more nonurologic complications (15.7 vs. $14.3 \%$; OR: 1.53 (95\% CI: $1.12-2.10), p=0.0077$ ). The major concerns for LPN were the higher observed rate of postoperative hemorrhage and urine leak that was accentuated by the larger tumors and less fit patients in the cohort undergoing OPN. ${ }^{6}$

With further refinements in technique, the results of minimally invasive $\mathrm{PN}$ have improved substantially during $15+$ years of refinement. ${ }^{3,8}$ With LPN, and more recently with robotic PN (RPN), ischemia times and complications have decreased substantially. ${ }^{3,9}$ When directly comparing similar cohorts undergoing LPN or RPN by the same surgeons, RPN was associated with shorter hospital stays (2.4 vs. 2.7 days $)$, shorter ischemic intervals $(19.7$ vs. $28.4 \mathrm{~min}$ ), and lower complication rates (8.6 vs. $10.2 \%$ ) in a multi-institutional study. ${ }^{10}$ Meta-analysis of multiple retrospective studies indicated that LPN and RPN provided comparable hospital stay and complication rates, with an advantage of shorter ischemia time for RPN even with greater tumor complexity in these cases. ${ }^{9} \mathrm{RPN}$ appears to have extended the reach of LPN, enabling PN to be performed in more complex scenarios and by a greater number of proficient surgeons.

The published evidence has suggested that RPN can likely be performed with a favorable risk-benefit profile relative to OPN for well-selected patients. ${ }^{3,11-13}$ This present report confirms these prior reports. ${ }^{14}$ Peyronnet et al. report in this issue of Annals of Surgical Oncology that RPN is associated with favorable perioperative outcomes when compared with OPN. Data were collected from 1800 
patients in this retrospective multicenter study, including 937 RPN and 863 OPN. The authors found that Clavien grade 3 or higher complications occurred less frequently with RPN (7.8 vs. $11 \%$ ) and after propensity-scoreweighted analysis, the odds ratio for complications with OPN was 2.11 (95\% CI: 1.53-2.91). The ischemia time was shorter (15.7 vs. $18.6 \mathrm{~min})$ with RPN and likely attributable to use of the early unclamping technique in this series. In multivariable analysis, this difference was not statistically significant and likely due to confounders. Hospital stay (4.7 vs. 10.1 days) and estimated blood loss ( 275 vs. $360 \mathrm{ml}$ ) also were lower with RPN. One potential limitation of MIPN is the surgical learning curve, which appears to be longer with LPN than with RPN. ${ }^{15-18}$ Most studies suggest that outcomes improve after a surgeon completes between 30 and 40 cases. The current work supports the notion that complication rates are higher during a surgeon's (or institution's) learning curve: major and hemorrhagic complications were nearly twofold higher for "PN performed during the first year after acquisition of the robot" $(p=0.02)$. Each surgeon and institution should consider the implications of this process on their patients.

RPN did not appear to compromise oncologic outcomes, because surgical approach did not have an association $(p=0.72)$ with cancer-specific survival (CSS), albeit at a median follow-up of 13 months. Prognostic factors for CSS were tumor size $(p=0.01)$ and Fuhrman grade $(p=0.007)$. Other reports have demonstrated similar findings, namely that tumor characteristics (not surgical approach: LPN vs. OPN) are the main predictors of recurrence at 10 years after PN. ${ }^{19}$

Strengths of the present work include the large sample size, statistically and clinically significant outcomes reported, and overall significance of the findings in the context of contemporary practice. The limitations include the retrospective and observational nature of the study and the short follow-up after RPN. With the wide integration of the Da Vinci surgical system for RPN, additional data from multiple centers and with more robust follow-up will no doubt confirm the validity of these findings. Robotic technology has made this challenging operation more accessible and more reproducible, such that the move to RPN as a reference standard for small renal masses appears to have occurred. The successful treatment of larger and more complex tumors with RPN indicates that it has the potential to permit more patients who stand to benefit from a kidney-sparing surgery to receive one with less morbidity.

ACKNOWLEDGMENT The corresponding author thanks the Betz Family Endowment for Cancer Research for their support. We would also like to thank Sabrina Noyes for administrative support in preparing and submitting the editorial.

\section{REFERENCES}

1. Campbell SC, Novick AC, Belldegrun A, et al. Guideline for management of the clinical T1 renal mass. J Urol. 2009;182:1271.

2. Liu JJ, Leppert JT, Maxwell BG, et al. Trends and perioperative outcomes for laparoscopic and robotic nephrectomy using the National Surgical Quality Improvement Program (NSQIP) database. Urol Oncol. 2014;32:473.

3. Wu Z, Li M, Liu B, et al. Robotic versus open partial nephrectomy: a systematic review and meta-analysis. PLoS One. 2014;9:e94878.

4. Gill IS, Desai MM, Kaouk JH, et al. Laparoscopic partial nephrectomy for renal tumor: duplicating open surgical techniques. J Urol. 2002;167:469.

5. Link RE, Bhayani SB, Allaf ME, et al. Exploring the learning curve, pathological outcomes and perioperative morbidity of laparoscopic partial nephrectomy performed for renal mass. $J$ Urol. 2005;173:1690.

6. Gill IS, Matin SF, Desai MM, et al. Comparative analysis of laparoscopic versus open partial nephrectomy for renal tumors in 200 patients. J Urol. 2003;170:64.

7. Gill IS, Kavoussi LR, Lane BR, et al. Comparison of 1,800 laparoscopic and open partial nephrectomies for single renal tumors. J Urol. 2007;178:41.

8. Simone G, Gill IS, Mottrie A, et al. Indications, techniques, outcomes, and limitations for minimally ischemic and off-clamp partial nephrectomy: a systematic review of the literature. Eur Urol. 2015;68:632.

9. Choi JE, You JH, Kim DK, et al. Comparison of perioperative outcomes between robotic and laparoscopic partial nephrectomy: a systematic review and meta-analysis. Eur Urol. 2015;67:891.

10. Benway BM, Bhayani SB, Rogers CG, et al. Robot assisted partial nephrectomy versus laparoscopic partial nephrectomy for renal tumors: a multi-institutional analysis of perioperative outcomes. J Urol. 2009;182:866.

11. Ficarra V, Minervini A, Antonelli A, et al. A multicentre matched-pair analysis comparing robot-assisted versus open partial nephrectomy. BJU Int. 2014;113:936.

12. Simhan J, Smaldone MC, Tsai KJ, et al. Perioperative outcomes of robotic and open partial nephrectomy for moderately and highly complex renal lesions. J Urol. 2012;187:2000.

13. Wu Z, Li M, Qu L, et al. A propensity-score matched comparison of perioperative and early renal functional outcomes of robotic versus open partial nephrectomy. PLoS One. 2014;9:e94195.

14. Peyronnet B, Seisen T, Oger E, et al. Comparison of 1800 robotic and open partial nephrectomies for renal tumors. Ann Surg Oncol. 2016. doi:10.1245/s10434-016-5411-0.

15. Hanzly M, Frederick A, Creighton T, et al. Learning curves for robot-assisted and laparoscopic partial nephrectomy. J Endourol. 2015;29:297.

16. Kapoor A. Laparoscopic partial nephrectomy: a challenging operation with a steep learning curve. Can Urol Assoc J. 2009:3:119.

17. Pierorazio PM, Patel HD, Feng T, et al. Robotic-assisted versus traditional laparoscopic partial nephrectomy: comparison of outcomes and evaluation of learning curve. Urology. 2011;78:813.

18. Mottrie A, De Naeyer G, Schatteman P, et al. Impact of the learning curve on perioperative outcomes in patients who underwent robotic partial nephrectomy for parenchymal renal tumours. Eur Urol 2010;58:127.

19. Lane BR, Campbell SC, Gill IS. 10-year oncologic outcomes after laparoscopic and open partial nephrectomy. J Urol. 2013;190:44. 